\title{
Fusion of Multispectral and Panchromatic Images Using Improved IHS and PCA Mergers Based on Wavelet Decomposition
}

\author{
María González-Audícana, José Luis Saleta, Raquel García Catalán, and Rafael García
}

\begin{abstract}
Since Chavez proposed the highpass filtering procedure to fuse multispectral and panchromatic images, several fusion methods have been developed based on the same principle: to extract from the panchromatic image spatial detail information to later inject it into the multispectral one. In this paper, we present new fusion alternatives based on the same concept, using the multiresolution wavelet decomposition to execute the detail extraction phase and the intensity-hue-saturation (IHS) and principal component analysis (PCA) procedures to inject the spatial detail of the panchromatic image into the multispectral one. The multiresolution wavelet decomposition has been performed using both decimated and undecimated algorithms and the resulting merged images compared both spectral and spatially. These fusion methods, as well as standard IHS-, PCA-, and wavelet-based methods have been used to merge Systeme Pour l'Observation de la Terre (SPOT) $4 \mathrm{XI}$ and SPOT $4 \mathrm{M}$ images with a ratio $4: 1$. We have estimated the validity of each fusion method by analyzing, visually and quantitatively, the quality of the resulting fused images. The methodological approaches proposed in this paper result in merged images with improved quality with respect to those obtained by standard IHS, PCA, and standard wavelet-based fusion methods. For both proposed fusion methods, better results are obtained when an undecimated algorithm is used to perform the multiresolution wavelet decomposition.
\end{abstract}

Index Terms-Decimated wavelet transform, intensity-hue-saturation (IHS) transform, image-fusion, multiresolution analysis, principal component analysis (PCA), undecimated wavelet transform.

\section{INTRODUCTION}

$\mathbf{F}$ OR OPTICAL remote sensing sensors, spatial and spectral resolutions are highly correlated factors. Given the design constraints of these sensors, there is an inverse relation between their spatial and spectral resolution. In general, sensors with high spectral resolution, characterized by capturing the radiance from the different land covers in a high number of bands of the electromagnetic spectrum, do not have an optimal spatial resolution, and vice-versa.

Manuscript received December 5, 2002; revised December 31, 2003. This work was supported in part by the Gobierno de Navarra under Grant 58/1999 and in part by the Comisión Interministerial de Ciencia y Tecnología under Grant AGL2000-0978.

M. González-Audícana and R. García are with the Department of Projects and Rural Engineering, Public University of Navarre, 31006 Pamplona, Spain (e-mail: maria.audicana@unavarra.es).

J. L. Saleta is with the Donal Bren School of Environmental Sciences and Management, University of California, Santa Barbara CA 93106-5131 USA (e-mail: saleta@bren.ucsb.edu).

R. G. Catalán is with the Department of Applied Mathematics, Public University of Navarre, 31006 Pamplona, Spain (e-mail: raquel.garcia@si.unavarra.es). Digital Object Identifier 10.1109/TGRS.2004.825593
The availability of high spectral and spatial resolution images is desirable when undertaking identification studies in areas with complex morphological structure such as urban areas, heterogeneous forested areas or agricultural areas with a high degree of plot subdivision. On one hand, a high spectral resolution eases the discrimination of land cover types. On the other hand, it is necessary a high spatial resolution to be able to accurately delimit the area occupied by each land cover type, as well as to locate the different terrain features and structures.

The fusion of multispectral (MS) and panchromatic (PAN) images, with complementary spectral and spatial characteristics, is becoming a promising technique to obtain images with high spatial and spectral resolution simultaneously.

Probably the most popular image-fusion methods are those based on the intensity-hue-saturation transformation (IHS) [1]-[4] and principal component analysis (PCA) [5]. The main drawback of these methods, frequently called "component substitution" methods [6], is the high distortion of the original spectral information that the resulting MS images present [5], [7], [8].

This modification of the spectral information during the fusion process is not acceptable when the resulting images are to be used to extract thematic information such as land uses/cover mapping though a spectral classification procedure.

Based on Schowengerdt's work [9], Chavez proposed a new fusion method that minimizes the above-mentioned problem: the highpass filtering (HPF) method [10], [11]. The main idea of this method is to extract from the PAN image the high-frequency information, which is related to spatial information, to later insert or inject it into the MS image previously expanded to match the PAN pixel size. This spatial information extraction is performed applying a highpass spatial filter to the PAN image, while the spatial information injection is performed adding, pixel by pixel, the filtered image that results from the extraction process to the MS one.

The HPF method could be considered the predecessor of an extended group of image fusion procedures based on the same principle: to extract from the PAN image the spatial detail information not present in the MS one, to later inject it into the latter, in a multiresolution framework [Amélioration de la Résolution Spatiale par Injection de Structures (ARSIS) concept] [12].

In the past few years, several researchers have proposed different PAN and MS image-fusion methods based on this concept, employing the discrete wavelet transform [7], [8], [12]-[14], Laplacian pyramid algorithms [15], [16], or "à trous" 
wavelet transforms [17], [18] to perform the detail extraction and injection processes.

In this paper, we present new fusion alternatives based on the same concept, using the multiresolution wavelet decomposition proposed by Mallat [19] to execute the detail extraction phase, and the IHS and PCA procedures to inject the spatial detail of the PAN image into the MS one. When the Mallat's algorithm is employed to perform the discrete wavelet decomposition of an image, a filtering and subsampling procedure is applied. This subsampling process could cause a loss of the lineal continuity of spatial details such as plot edges, railways, or roads. To avoid this problem, a redundant discrete wavelet decomposition scheme (without subsampling) will be applied.

We have used these fusion methods, as well as standard IHS-, PCA-, and wavelet-based methods, to merge Systeme Pour l'Observation de la Terre (SPOT) 4 XI and SPOT $4 \mathrm{M}$ images of an agricultural area with heterogeneous field structure, both in size and geometry.

We have determined the validity of each fusion method by analyzing the spectral quality [20] and also the spatial quality of the resulting fused images.

In order to assess the quality of the resulting images, they should be compared with the image that the MS sensor would theoretically collect if it had the same spatial resolution as the PAN [20]. Since these images do not exist, we decided to work with spatially degraded images. The spatial resolution ratio between the PAN and MS sensors installed in the recently launched IKONOS, QuickBird, or SPOT 5 satellites is $1: 4$, so the SPOT $4 \mathrm{M}$ image degraded to $20 \mathrm{~m}$ was merged with the $\mathrm{XI}$ degraded to $80 \mathrm{~m}$.

\section{STANDARD IHS AND PCA MERGERS}

Both IHS and PCA mergers are based on the same principle: to separate most of the spatial information of an MS image from its spectral information by means of linear transforms.

The IHS transform separates the spatial information of the MS image as the intensity (I) component. In the same way, PCA separates the spatial information of the MS image into the first principal component $\left(\mathrm{PC}^{1}\right)$ [21].

\section{A. Standard IHS Merger}

The widespread use of the IHS transform to merge images is based on its ability to separate the spectral information of an RGB composition in its two components $\mathrm{H}$ and $\mathrm{S}$, while isolating most of the spatial information in the I component [22].

Several algorithms have been developed that allow the conversion of the color values (RGB) into values of intensity, hue, and saturation [23]-[26]. Whatever algorithm is chosen, the IHS transform is always applied to an RGB composite. This implies that the fusion will be applied to groups of three bands of the MS image. As a result of this transformation, we obtain the new intensity, hue, and saturation components. The PAN image then replaces the intensity image. Before doing this, and in order to minimize the modification of the spectral information of the fused MS image with respect to the original MS image, the histogram of the PAN image is matched with that of the intensity image. Applying the inverse transform, we obtain the fused
RGB image, with the spatial detail of the PAN image incorporated into it.

\section{B. Standard PCA Merger}

Most MS sensors collect information in adjacent bands of the electromagnetic spectrum. This generally implies detecting redundant information, since many land covers tend to behave in a similar fashion in neighboring regions of the spectrum.

In this context, PCA allows to synthesize the original bands creating new bands, the principal components, which pick up and reorganize most of the original information.

In general, the first principal component $\left(\mathrm{PC}^{1}\right)$ collects the information that is common to all the bands used as input data in the PCA, i.e., the spatial information, while the spectral information that is specific to each band is picked up in the other principal components [21]. This makes PCA a very adequate technique when merging MS and PAN images.

In this case, all the bands of the original MS image constitute the input data. As a result of this transformation, we obtain noncorrelated new bands, the principal components.

The $\mathrm{PC}^{1}$ is substituted by the PAN image, whose histogram has previously been matched with that of $\mathrm{PC}^{1}$. Finally, the inverse transformation is applied to the whole dataset formed by the modified PAN image and the $\mathrm{PC}^{2} \ldots \mathrm{PC}^{\mathrm{n}}$, obtaining that way the new fused bands with the spatial detail of PAN image incorporated into them.

\section{IMPROVED IHS AND PCA MERGERS}

Standard IHS and PCA methods provide an adequate imagefusion framework when the PAN image is highly correlated with the I or PC ${ }^{1}$ components of the MS image to be fused with.

As both the I and the $\mathrm{PC}^{1}$ components are obtained as linear combinations of the different bands of the MS image, a high correlation between these and the PAN image can only be possible if the bandwidth of the latter covers the entire range of bandwidths of all the MS original bands, and also if both PAN and MS images are captured at the same time.

If any of these two conditions is not met, the assumption about the separation of the spectral and spatial content of an MS image made when those mergers are used could result in low-quality merged images. In fact, when IHS and PCA transforms are applied, the spectral and spatial information of the MS image is not completely separated. The $\mathrm{H}$ and $\mathrm{S}$, or $\mathrm{PC}^{2} \ldots \mathrm{PC}^{\mathrm{n}}$, components carry some spatial information associated with them, in the same manner that the I and $\mathrm{PC}^{1}$ images have certain spectral information associated. Therefore, the images fused using these methods tend to present more spatial detail information than what the MS image would have if it had been collected with the spatial resolution of the PAN image. Besides, the spectral information of these merged images also differs significantly from what the MS image would have if it had been collected at the resolution of the PAN image.

The spectral and spatial quality of the merged images would improve substantially if instead of replacing the I or $\mathrm{PC}^{1}$ components with the PAN image, we could introduce in these components just the spatial detail of the PAN image that is missing 
in the MS. This is the central idea of the improved IHS and PCA mergers.

The detail information of the PAN image that corresponds to structures or features with a size between the spatial resolution of the PAN image and that of the MS one is extracted using the multiresolution wavelet decomposition (MWD) proposed by Mallat in a decimated or undecimated way.

\section{A. Multiresolution Wavelet Decomposition}

Multiresolution analysis allows to decompose images into a set of new images with coarser and coarser spatial resolution (approximation images). Associated to the latter, the wavelet transform describes the difference in the information of two successive approximation images (wavelet coefficient images). This information difference corresponds to structures with a size between the two resolutions of the successive approximation images. For further information on this topic, see [27] and [28].

The discrete approach of the wavelet transform can be performed using several different algorithms

1) Mallat's Decimated Algorithm [19]: It is a fast wavelet transform algorithm based on a multiresolution dyadic scheme that allows to decompose an image $A_{2^{j}}$ at a resolution $2^{j}$, into an approximation image $A_{2^{j-1}}$ at a resolution $2^{j-1}$ and three wavelet coefficient images $D H_{2^{j-1}}, D V_{2^{j-1}}$, and $D D_{2^{j-1}}$, which pick up, respectively, the horizontal, vertical, and diagonal detail that is lost between the images $A_{2^{j}}$ and $A_{2^{j-1}}$. If the original image $A_{2^{j}}$ has $C$ columns and $R$ rows, the approximation and the wavelet coefficient images obtained applying this multiresolution decomposition have $C / 2$ columns and $R / 2$ rows.

The computation of the approximation and the detail coefficients is accomplished with a pyramidal scheme based on convolutions along rows and columns with one-dimensional filters followed by a subsampling or decimation operation [19].

When the MWD process is inverted, the original image $A_{2^{j}}$ can be reconstructed exactly from an approximation image $A_{2^{j-1}}$ and the wavelet coefficients $D H_{2^{j-1}}, D V_{2^{j-1}}$, and $D D_{2^{j-1}}$ applying an upsampling or oversamplig process followed by filtering.

Both decomposition and reconstruction filters have to be quadrature mirror filters [29] in order to satisfy constraints inherent to a perfect reconstruction of the original image $A_{2^{j}}$.

2) Undecimated Algorithm: This algorithm is based on the idea of no decimation. It is a redundant wavelet transform algorithm based on a multiresolution dyadic scheme accomplished not with a pyramidal scheme but with a parallelepipedic scheme. The original image $A_{2^{j}}$ at a resolution $2^{j}$ is decomposed into an approximation image $A_{2^{j-1}}$ at a resolution $2^{j-1}$ and three wavelet coefficient images, $D H_{2^{j-1}}$, $D V_{2^{j-1}}$, and $D D_{2^{j-1}}$ which pick up the detail information that is lost between the images $A_{2^{j}}$ and $A_{2^{j-1}}$. In contrast to the decimated, the undecimated algorithm allows a shift-invariant discrete wavelet decomposition. All the approximation and wavelet coefficient images obtained by applying this algorithm have the same number of columns and rows as the original image thus such decomposition is highly redundant. A detailed discussion on undecimated algorithms can be found in [30] and [31].
The practical implementation of this algorithm is similar to that of the Mallat's decimated one [19] but in this case, the subsampling or decimation operation is suppressed and the decomposition and reconstruction filters are upsampled inserting zeros between its coefficients with a step $2^{j-1}$.

The number of coefficients of these filters and the value of each coefficient depends on the Mother Wavelet function used in this analysis. In this paper, we have used the Daubechies fourcoefficient wavelet basis [32] to perform both the decimated and the undecimated algorithms.

\section{B. Improved IHS and PCA Mergers, Based on MWD}

Fig. 1 outlines the general procedure to fuse MS and PAN images, with a spatial resolution ratio of $1: 2$, using the PCA improved method based on the decimated or undecimated MWD. These are the steps followed.

1) Coregister both images and resample the MS image to make its pixel size equal to that of the PAN, in order to get perfectly superposable images.

2) Apply the IHS transform or PCA to the MS image and obtain the I or $\mathrm{PC}^{1}$ component.

3) Generate a new PAN image whose histogram matches that of the I or $\mathrm{PC}^{1}$ image.

4) Apply the decimated or undecimated wavelet decomposition to the I or $\mathrm{PC}^{1}$ image and to the corresponding histogram-matched PAN $\left(\mathrm{PAN}_{\mathrm{xx}}\right)$, using the Daubechies four-coefficient wavelet. From each I, $\mathrm{PC}^{1}$, or PANxx image decomposition, four half-resolution images are obtained $(J=1)$.

The first one, the approximation image, is a low-frequency version of the I, $\mathrm{PC}^{1}$, or PAN image, and the other three images $\left(H D^{x x}, V D^{x x}, D D^{x x}\right)$, the wavelet coefficient zero-mean images, which pick up the spatial detail information between the original image at resolution "P" and the approximation image at resolution "P/2."

5) Inject the detail coefficients of the PAN image that pick up the spatial detail information present in this image and missing in the MS one, into the I or $\mathrm{PC}^{1}$ image through the inverse MWD process. The transformation model used in this paper to generate the high-frequency coefficients to be injected into the I or $\mathrm{PC}^{1}$ image is the identity model [33], [34]. In order to take into account the spectral differences in the detail information between the PAN and I or $\mathrm{PC}^{1}$ images, the histogram of the former must previously match that of the I or $\mathrm{PC}^{1}$ [step 3)].

6) Insert the spatial information of the PAN image into the MS one through the inverse IHS transform or inverse PCA.

The result of this process is a merged image with high spatial resolution that keeps the spectral information of the original MS image, as will be demonstrated in the following section.

\section{EXPERIMENTAL RESULTS}

A couple of SPOT $4 \mathrm{XI}$ and SPOT $4 \mathrm{M}$ images collected in November 1999 and covering an agricultural irrigated area of Navarre, Spain, were used as MS and PAN test images (Fig. 2) 


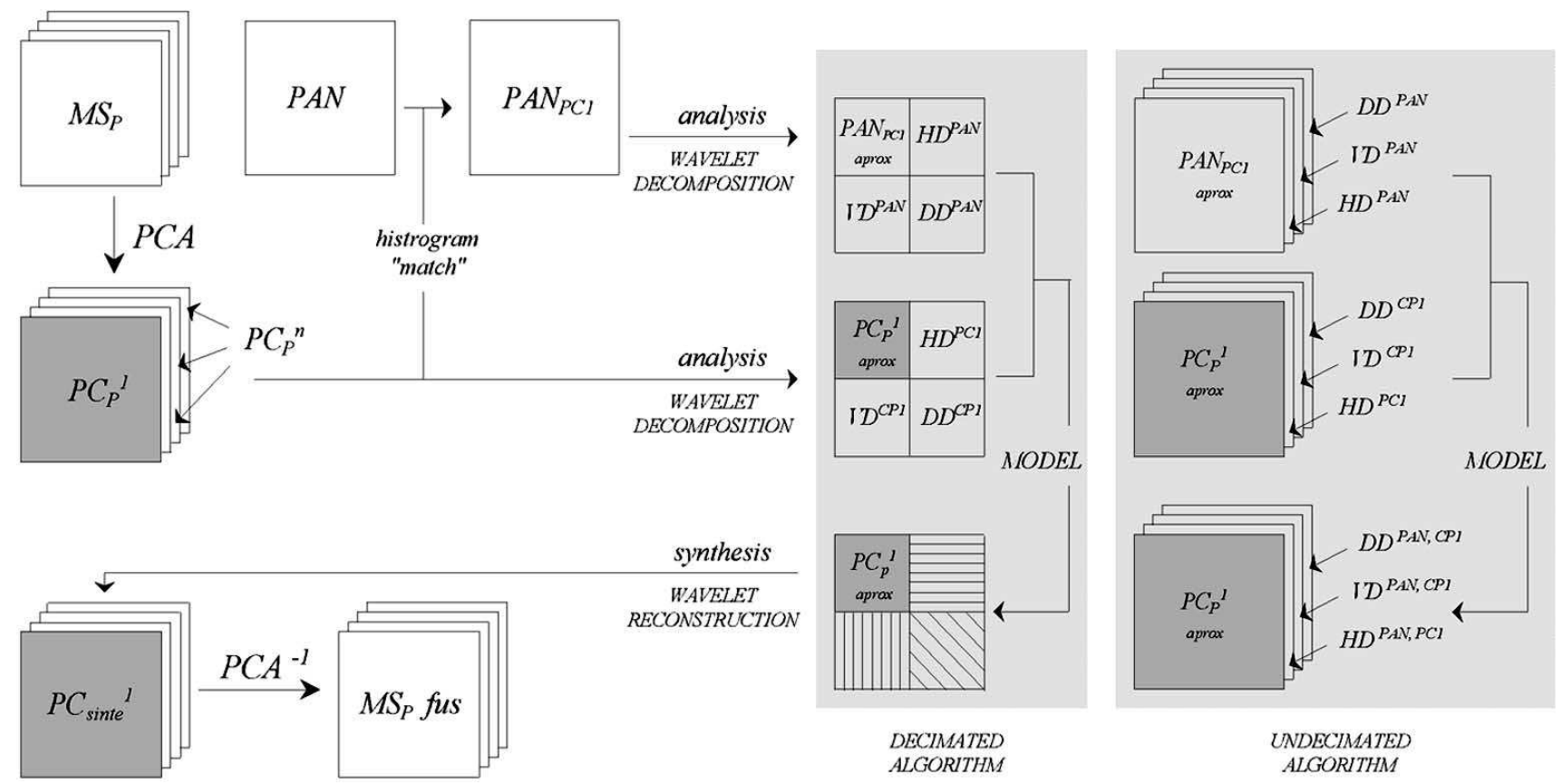

Fig. 1. dWPC and udWPC image-fusion methods. General application diagram for the fusion of MS and PAN images with a spatial resolution ration of 2 : 1.

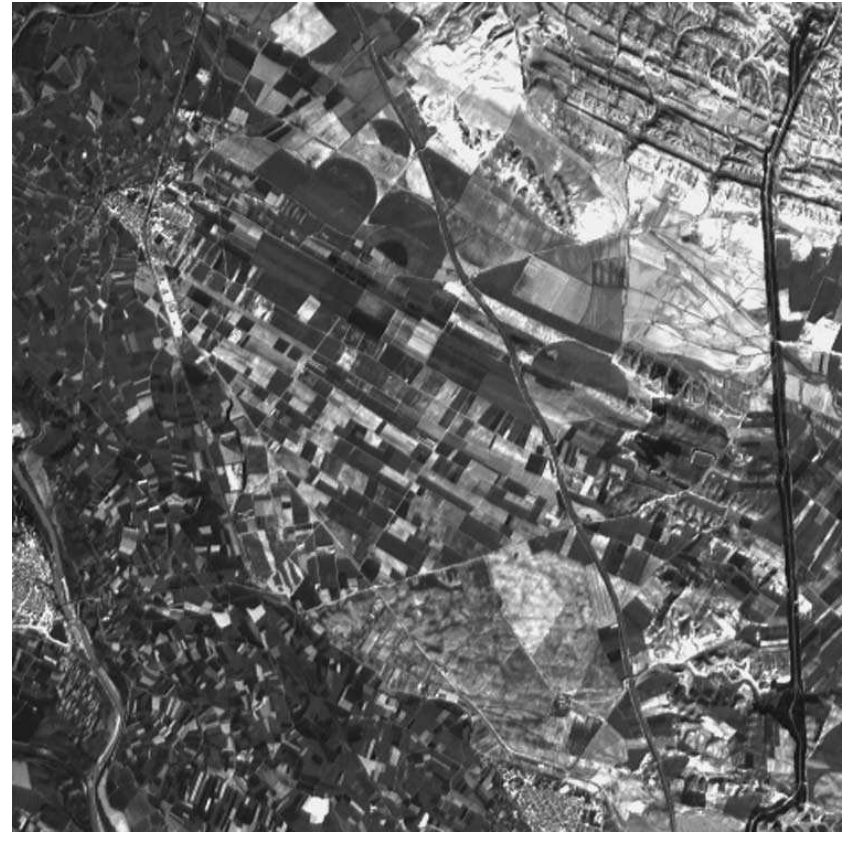

Fig. 2. SPOT $4 \mathrm{M}$ image at $20 \mathrm{~m}$ corresponding to the test area $(512 \times 512)$.

to evaluate and compare the performance of the following fusion methods:

- Standard IHS, using the Smith's triangle model [23] to convert the RGB values into intensity, hue, and saturation, and standard PCA.

- Standard MWD-based method [7], [8], [12], [13], injecting the spatial detail of the PAN image into each band of the MS one, using the Daubechies four-coefficient wavelet basis to perform both the decimated ( $\mathrm{dW}$ ) and undecimated ( $\mathrm{udW})$ alternatives.
- Improved IHS and PCA: MWD-based method, injecting the spatial detail of the PAN image into the I or PC ${ }^{1}$ images, using the Daubechies four-coefficient wavelet basis to perform both the decimated (dWI, dWPC) and undecimated (udWI, udWPC) algorithms.

The spatial resolution of the SPOT 4 XI (multispectral) and SPOT $4 \mathrm{M}$ (monospectral) images is, respectively, 20 and $10 \mathrm{~m}$. The high spatial resolution MS images obtained applying any of the image fusion methods described earlier would have a real spatial resolution similar to that of the PAN image, in our case $10 \mathrm{~m}$.

In order to assess the quality of the merged images, they should be compared with the image that the SPOT 4 HRVIR sensor would theoretically collect in multispectral mode if it had a spatial resolution of $10 \mathrm{~m}$. Since these images do not exist, we worked with spatially degraded images. The MS and PAN SPOT images were degraded to 80 and $20 \mathrm{~m}$, respectively, to simulate the fusion of MS and PAN with a spatial resolution ratio of four.

Merged images obtained by different methods have a spatial resolution of $20 \mathrm{~m}$, so the goodness of each image-fusion method can be evaluated by comparing the resulting merged images with the SPOT 4 XI original one.

This comparison is based on spectral and spatial characteristics, and is done both visually and quantitatively.

\section{A. Spectral Quality of the Merged Images}

The spectral quality of the merged images will be evaluated by comparing their spectral information to that of the original SPOT 4 XI image [12], [20]. This comparison is performed both visually and quantitatively using the following indicators:

- Correlation coefficient (CC) between the original and the merged images. It should be as close to 1 as possible.

- Difference between the means of the original and the merged images (bias), in radiance as well as its value rel- 
TABLE I

Value of the Different Parameters Analyzed to Estimate the Spectral Quality of the Merged Images

\begin{tabular}{lccccccccccc}
\hline \hline & & Initial & IHS & PCA & dW & udW & dWI & udWI & dWPC & udWPC & Ideal \\
\hline \multirow{2}{*}{ Correlation } & $\mathrm{X} 1$ & 0.926 & 0.965 & 0.964 & 0.977 & 0.982 & 0.977 & 0.980 & 0.978 & 0.982 & 1 \\
coefficient & $\mathrm{X} 2$ & 0.938 & 0.982 & 0.967 & 0.983 & 0.987 & 0.984 & 0.985 & 0.983 & 0.987 & 1 \\
& $\mathrm{X} 3$ & 0.925 & 0.790 & 0.919 & 0.849 & 0.875 & 0.884 & 0.908 & 0.920 & 0.932 & 1 \\
& $\mathrm{X} 4$ & 0.917 & 0.939 & 0.935 & 0.941 & 0.951 & 0.947 & 0.951 & 0.950 & 0.955 & 1 \\
\hline \multirow{2}{*}{$\begin{array}{l}\text { Mean diffiderence } \\
\text { relative to original }\end{array}$} & $\mathrm{X} 1$ & 0.00 & -0.16 & 0.00 & 0.00 & 0.00 & 0.00 & 0.00 & 0.00 & 0.00 & 0 \\
mean (\%) & $\mathrm{X} 3$ & 0.01 & 1.43 & 0.01 & 0.02 & 0.02 & 0.01 & 0.01 & 0.01 & 0.01 & 0 \\
\hline SDD relative & $\mathrm{X} 4$ & 0.00 & 0.18 & 0.02 & 0.04 & 0.03 & 0.01 & 0.01 & 0.01 & 0.01 & 0 \\
to original & $\mathrm{X} 1$ & 9.46 & 6.67 & 6.78 & 5.44 & 4.69 & 5.44 & 5.16 & 5.27 & 4.74 & 0 \\
mean (\%) & $\mathrm{X} 2$ & 11.80 & 6.91 & 8.43 & 6.34 & 5.61 & 6.31 & 6.02 & 6.34 & 5.61 & 0 \\
& $\mathrm{X} 4$ & 11.63 & 8.79 & 9.04 & 8.45 & 7.59 & 7.98 & 7.64 & 7.80 & 7.25 & 0 \\
\hline RASE (\%) & & 11.8 & 13.9 & 11.5 & 11.2 & 10.6 & 10.5 & 9.8 & 9.3 & 8.9 & 0 \\
\hline ERGAS & & 2.61 & 2.82 & 2.53 & 2.43 & 2.19 & 2.22 & 2.10 & 2.02 & 1.91 & 0 \\
\hline \hline
\end{tabular}

ative to the mean of the original image (RM). The smaller these differences are, the better the spectral quality of the merged image. They should be as close to 0 as possible.

- Standard deviation of the difference image (SDD), relative to the mean of the original image, expressed as percentage. The lower the value of this parameter, the better the spectral quality of the merged image.

These parameters allow us to determine the difference in spectral information between each band of the merged image and of the original image.

In order to estimate the global spectral quality of the merged images, we have used the following parameters.

- The relative average spectral error (RASE) index [12] expressed as a percentage. It characterizes the average performance of the method in the spectral bands considered

$$
\operatorname{RASE}=\frac{1}{M} \sqrt{\frac{1}{N} \sum_{i=1}^{N} \operatorname{RMSE}^{2}\left(B_{i}\right)}
$$

where $M$ is the mean radiance of the $N$ spectral bands $\left(B_{i}\right)$ of the original MS image, and the RMSE is the root mean square error computed in following the expression:

$$
\operatorname{RMSE}^{2}\left(B_{i}\right)=\operatorname{bias}^{2}\left(B_{i}\right)+\operatorname{SDD}^{2}\left(B_{i}\right) .
$$

- The erreur relative globale adimensionnelle de synthèse (ERGAS) index (or relative global dimensional synthesis error) in the fusion [35]:

$$
\text { ERGAS }=100 \frac{h}{l} \sqrt{\frac{1}{N} \sum_{i=1}^{N}\left(\frac{\operatorname{RMSE}^{2}\left(B_{i}\right)}{M_{i}^{2}}\right)}
$$

where $h$ is the resolution of the high spatial resolution image and $l$ the resolution of the low spatial resolution image and $M^{i}$ the mean radiance of each spectral band involved in the fusion

The lower the value of the RASE and ERGAS indexes, the higher the spectral quality of the merged images.
In Table I, we show the values of the parameters and quality indexes resulting from the comparison of the merged images obtained when applying the different fusion methods described and the original SPOT MS image.

In order to quantify the effect that fusions have over the initial MS image ( $80 \mathrm{~m}$ per pixel, spatially degraded image), we show in the first column the values of the different parameters obtained when this degraded image was compared with the original MS image (20 m per pixel). Therefore, this first column reflects the situation before the fusion, while the last column reflects the situation that ideally should be reached after the fusion.

Lower CC or higher RM, SDD, RASE, or ERGAS values than those showed in the first column indicate that the analyzed image-fusion procedure tends to modify the spectral information of the initial MS image, while higher CC and lower RM, SDD, RASE, or ERGAS values imply that the fusion method used allows a high-quality transformation of the MS content when increasing the spatial resolution.

To ease the comparison of the different fusion methods, we have displayed the values of the spectral CC and of the ERGAS index of the merged images using bar diagrams (Fig. 3).

It can be observed that the fusion method that results in images of the least spectral quality is the one based on IHS transforms. Although the $\mathrm{CC}$ values of the $\mathrm{X} 1, \mathrm{X} 2$, and $\mathrm{X} 4$ bands are higher after the fusion than before, the $\mathrm{CC}$ value corresponding to the $\mathrm{X} 3$ band is much lower after the fusion. Besides, the value of the ERGAS index is higher in the merged image than in the initial one, indicating that along the merging process the spectral information of the former has been modified significantly.

As we already mentioned in the first section of this paper, there are several algorithms that convert the RGB values into intensity, hue, and saturation. We chose the algorithm based on the Smith's triangle model [23], which considers the intensity as the average of the three RGB values, for this was the one that offered the best relative results when applied to image fusion [17], [36].

One of the disadvantages of this fusion method is that it can only be applied to three-band RGB compositions. In the 


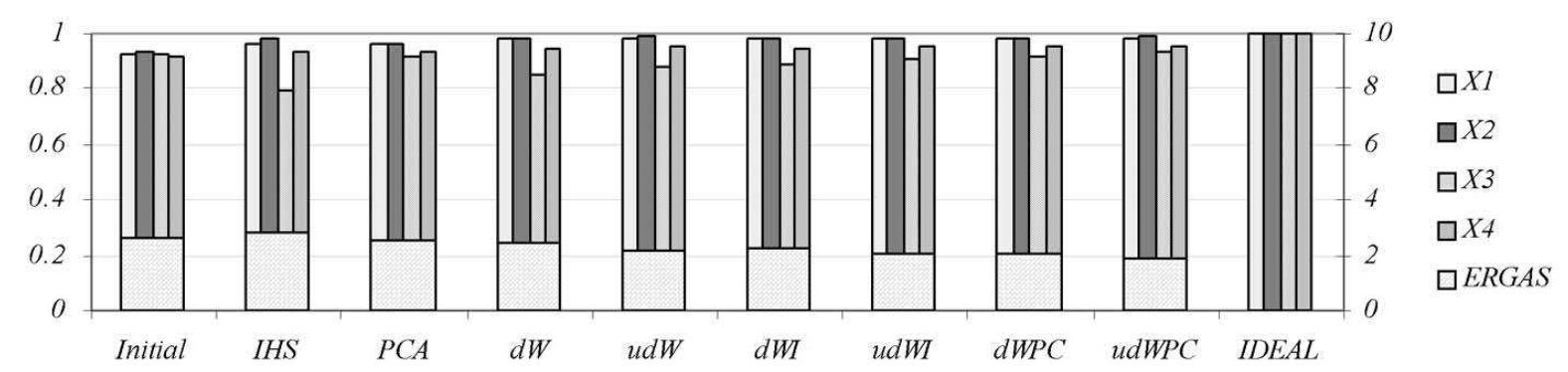

Fig. 3. Graphical representation of the values of the spectral correlation coefficient and of the ERGAS index corresponding to the merged images of "Villafranca."

case of SPOT 4 images, and for the purpose of comparing this method with other fusion methods, all the process described in Section II-A was repeated for the four possible RGB compositions of the initial MS image. This implies that for each spectral band we obtain three merged bands coming from the different RGB compositions. The final merged image is formed by the merged bands that have the highest spectral correlation with the respective spectral bands of the original SPOT 4 XI image.

The low spectral quality of the merged images obtained using IHS standard merger is obvious when the false color compositions of the merged images [Fig. 4(d)] are visually compared with the compositions of the initial MS images [Fig. 4(a)] and those of the original MS images [Fig. 4(c)]. It is noticeable the color variation in many of the fields and in the water pond.

Globally, the standard PCA method performs better than IHS. Although the spectral quality of X1, X2, and X4 bands is significantly higher after the fusion than before, the CC, RM and SDD values are worse for the X3 merged band than for the X3 initial band. This indicates that the image-fusion procedure has modified the spectral information of this band when increasing its spatial content.

dWI/udWI and dWPC/udWPC methods provide merged images of higher spectral quality than the standard IHS and PCA methods, respectively. The CC, RM, and SDD values for each band are better when improved IHS or PCA mergers are used instead of standard IHS or PCA mergers. In the same way, the RASE and ERGAS values for the dWI/udWI and for the dWPC/udWPC are lower than those for the standard IHS and PCA.

It can be observed in Fig. 4(g), (j), (h), and (k) how the merged images obtained applying these fusion methods have a very high spectral quality, as the colors of the agricultural fields, noncultivated areas, and water pond are the same as those of the original SPOT 4 XI image [Fig. 4(c)].

When applying fusion methods based on the IHS transform, the same amount of spatial detail gets inserted in all the bands of the MS image involved in the transform, independently of the correlation between these bands and the PAN image. The injection, in this case, of nonreal information into the NIR band results in a merged band of poor spectral quality.

The use of a very narrow PAN band, as that of the SPOT $4 \mathrm{M}$ mode, is more favorable to PCA than to IHS. However, if the bandwidth of the PAN sensor covers the entire range of bandwidths of all the MS original bands (i.e., IKONOS and QuickBird PAN sensor), the results obtained applying
IHS-based methods could be better than those obtained applying PCA-based methods [37].

Both band by band as well as globally, the spectral quality of the merged images obtained applying dWI/dWPC and udWI/udWPC is higher than that obtained applying $\mathrm{dW}$ and udW, respectively. When the latter methods are used, the detail information of the PAN image is injected into each MS band so the detail introduced, in this case, into the whole XI image is four times that injected into the same image when the improved IHS or PCA mergers are used. This redundant detail incorporation could explain the lower spectral quality of the dW/udW merged images.

\section{B. Spatial Quality of the Merged Images}

The main difference between the decimated and undecimated fusion algorithms is the presence or absence of subsampling when the MWD is performed. This subsampling operation, separately applied through rows and columns, causes a loss of linear continuity in spatial features such as edges, and the appearance of artifacts in those structures with neither horizontal nor vertical directions [Fig. 4(f)].

To provide a better view of the presence of artifacts, the spatial detail of the SPOT $4 \mathrm{M}$ original image was extracted applying a second level wavelet decomposition, using both decimated and undecimated algorithms. Fig. 5 shows these zero-mean images, biased and stretched for display purpose. As expected [16], [38], those artifacts are missing when the undecimated algorithm is applied, as can be observed in Fig. 4(i), (m), and Fig. 5. The use of the undecimated algorithm is justified not only because of the higher spatial quality but also because of a higher spectral quality of the merged images with respect to the same decimated methods, as can be derived from the RASE and ERGAS values in Table I.

In any case, the presence of artifacts is masked in the dWI and dWPC merged images because the spatial detail of the PAN image is injected just once into the whole MS image.

A good fusion method must allow the addition of a high degree of the spatial detail of the PAN image to the MS image. The addition of this spatial detail is evident for all the merged images when these are visually compared to the initial MS.

To evaluate this spatial detail addition, we used the procedure proposed by Zhou [12]. The PAN and merged images are filtered using the Laplacian filter

$$
\left|\begin{array}{ccc}
-1 & -1 & -1 \\
-1 & 8 & -1 \\
-1 & -1 & -1
\end{array}\right| .
$$




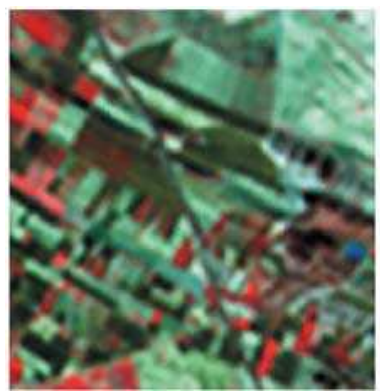

(a)

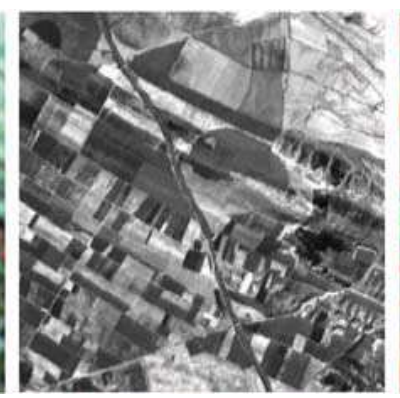

(b)

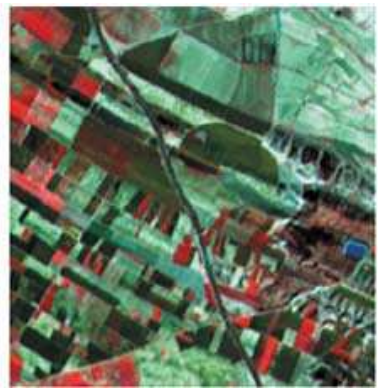

(c)

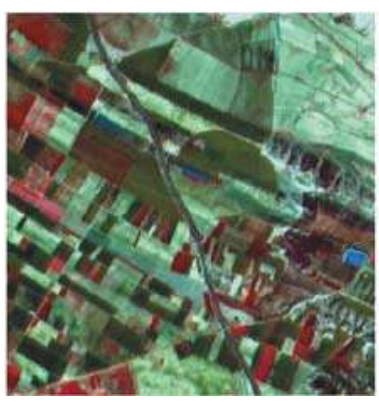

(d)

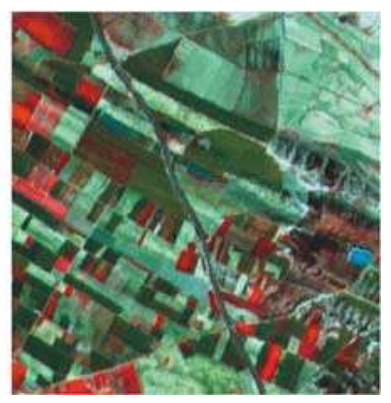

(e)

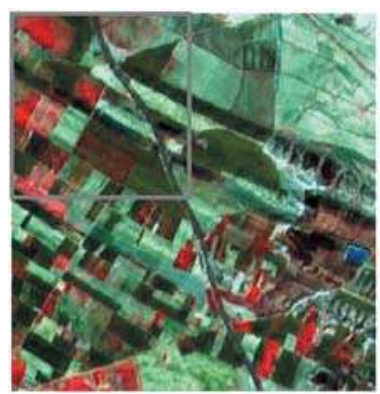

(f)

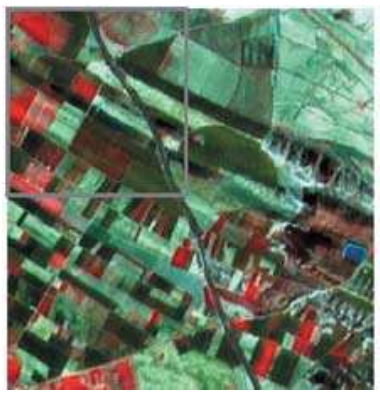

(i)

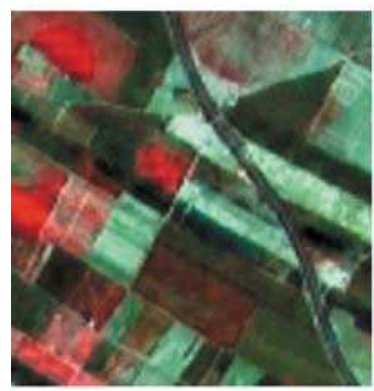

(1)

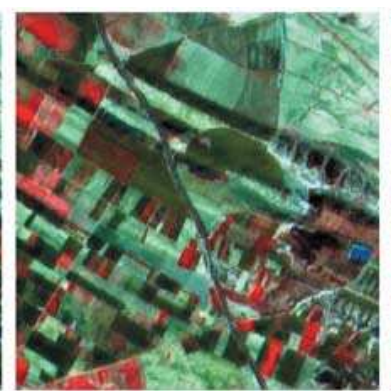

(g)

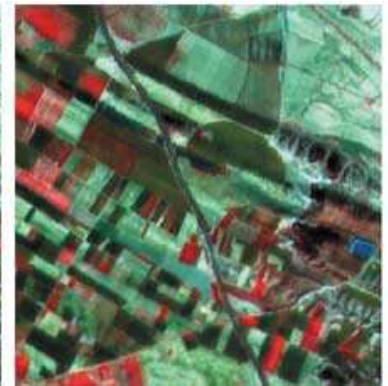

(j)

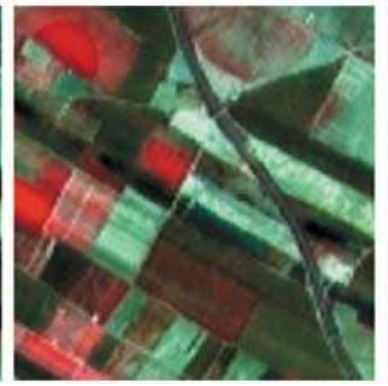

(m)

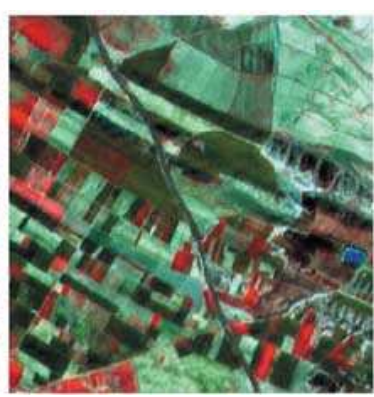

(h)

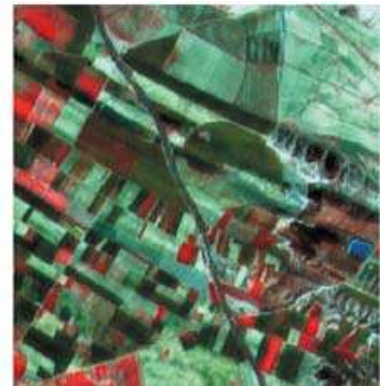

(k)

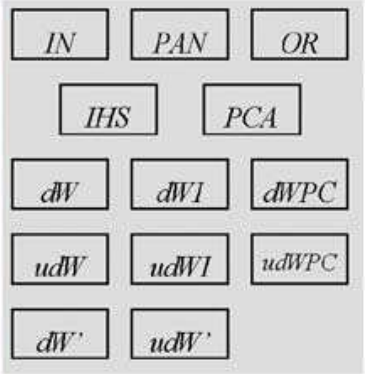

(n)

Fig. 4. (a) IN: detail from initial SPOT XI $(80 \mathrm{~m}$ resampled to $20 \mathrm{~m})(200 \times 200)$. (b) PAN: SPOT $4 \mathrm{M}(20 \mathrm{~m})$. (c) OR: original SPOT XI. (d) IHS-merged $(20 \mathrm{~m})$. (e) PCA-merged $(20 \mathrm{~m})$. (f) dW-merged $(20 \mathrm{~m})$. (g) dWI-merged (20 m). (h) dWPC-merged (20 m). (i) uDW-merged (20 m). (j) uDWI-merged (20 m). (k) udWPC-merged $(20 \mathrm{~m})$. (l) $\mathrm{dW}^{\prime}$ : detail from $(\mathrm{f})(100 \times 100)$. $(\mathrm{m}) \mathrm{udW}^{\prime}$ : detail from (i). 


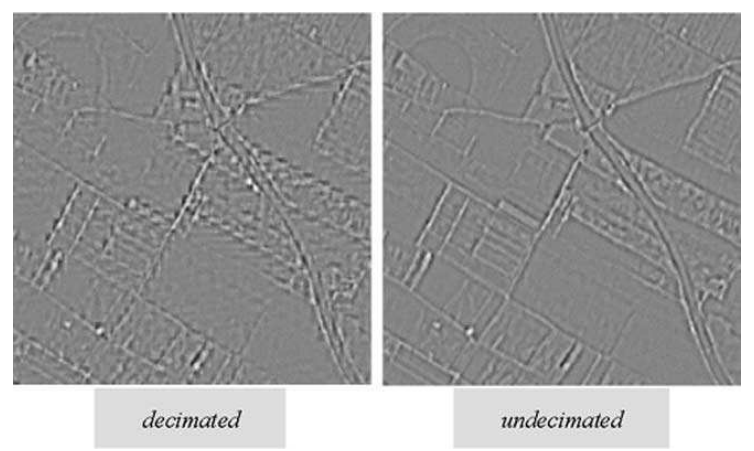

Fig. 5. Spatial detail of the SPOT $4 \mathrm{M}$ original image $(10 \mathrm{~m})$ extracted applying a second-level wavelet decomposition using both decimated and undecimated algorithms.

A high correlation between the merged filtered image and the PAN filtered one indicates that most spatial information of the PAN image has been incorporated during the fusion process. Although the correlation values ( $\mathrm{sCC}$ ) are low for the initial XI image $(0.20,0.21,0.06$, and 0.17 , respectively), each merged image presents sCC values higher than 0.85 for all bands. When the standard IHS and PCA mergers are used, the PAN image replaces the I or $\mathrm{PC}^{1}$ images, so the whole spatial detail information of this image is present in the resulting merged images. This explains the high sCC values corresponding to the standard IHS and PCA merged images. The lowest $\mathrm{SCC}$ values correspond to the udWI and udWPC merged images, in which only the spatial detail of the PAN image not present into the MS one was injected.

If we analyze visually the color compositions shown in Fig. 4, it is difficult to determine which fusion method gives images of better spatial quality (except for those based on the Mallat's decimated algorithm), but it is obvious that the spatial detail of the merged images has improved when compared with the initial MS image.

\section{CONCLUSION}

The alternative image-fusion methodological approaches presented in this paper, based on the IHS transformation and PCA using the MWD, allow to obtain merged images of higher quality than those obtained applying the IHS and PCA standard mergers. This higher quality is due to a selective incorporation into the multispectral image of just the spatial detail of the panchromatic image missing in the former, instead of performing a whole substitution. In addition, the injection of spatial detail extracted from the PAN image into the MS one just once when these methods are used results in images of higher spectral quality than those obtained applying standard wavelet-based merging methods where each MS band is fused with the PAN image separately.

As expected, artifacts are not detected in merged images when a translation-invariant undecimated algorithm is used to perform the MWD.

In the particular case of SPOT 4 images fusion, where the spectral bandwidth of the M sensor mode does not overlap with the entire range of bandwidths of all the XI bands, the methods based on PCA lead to better results than those based on the IHS transformation.

\section{REFERENCES}

[1] R. Haydn, G. W. Dalke, J. Henkel, and J. E. Bare, "Applications of the IHS color transform to the processing of multisensor data and image enhancement," in Proc. Int. Symp. Remote Sensing Arid and Semi-Arid Lands, Cairo, Egypt, 1982, pp. 559-616.

[2] R. Welch and M. Ehlers, "Merging multiresolution SPOT HRV and Landsat TM data," Photogramm. Eng. Remote Sens., vol. 53, pp. 301-303, Mar. 1987.

[3] J. Carper, T. M. Lillesand, and R. W. Kiefer, "The use of intensity-huesaturation transformations for merging SPOT panchromatic and multispectral image data," Photogramm. Eng. Remote Sens., vol. 56, pp. 459-467, Apr. 1990.

[4] M. Ehlers, "Multisensor image fusion techniques in remote sensing," ISPRS J. Photogramm. Remote Sens., vol. 46, pp. 19-30, 1991.

[5] P. S. Chavez, J. Stuart, C. Sides, and J. A. Anderson, "Comparison of three different methods to merge multiresolution and multispectral data: Landsat TM and SPOT panchromatic," Photogramm. Eng. Remote Sens., vol. 57, pp. 259-303, Mar. 1991.

[6] V. K. Shettigara, "A generalized component substitution technique for spatial enhancement of multispectral images using a higher resolution dataset," Photogramm. Eng. Remote Sens., vol. 58, pp. 561-567, May 1992.

[7] B. Garguet-Duport, J. Girel, J. M. Chasseny, and G. Pautou, "The use of multiresolution analysis and wavelet transform for merging SPOT panchromatic and multispectral image data," Photogramm. Eng. Remote Sens., vol. 62, pp. 1057-1066, Sept. 1996.

[8] J. Zhou, D. L. Civco, and J. A. Silander, "A wavelet transform method to merge Landsat TM and SPOT panchromatic data," Int. J. Remote Sens., vol. 19 , no. 4 , pp. $743-757,1998$.

[9] R. A. Schowengerdt, "Reconstruction of multispatial, multispectral image using spatial frequency contents," Photogramm. Eng. Remote Sens., vol. 46, pp. 1325-1334, Oct. 1980.

[10] P. S. Chavez, "Digital merging of Landsat TM and digitized NHAP data for $1: 24.000$ scale image mapping," Photogramm. Eng. Remote Sens., vol. 52, pp. 1637-1646, Oct. 1986.

[11] P. S. Chavez and J. Bowell, "Comparison of the spectral information content of Landsat Thematic Mapper and SPOT for three different sites in Phoenix, Arizona region," Photogramm. Eng. Remote Sens., vol. 54, pp. 1699-1708, Dec. 1988.

[12] T. Ranchin and L. Wald, "Fusion of high spatial and spectral resolution images: The ARSIS concept and its implementation," Photogramm. Eng. Remote Sens., vol. 66, pp. 49-61, Jan. 2000.

[13] D. A. Yocky, "Image merging and data fusion by means of the discrete two-dimensional wavelet transform," J. Opt. Soc. Amer. A., vol. 12, pp. 1834-1841, Sep. 1995.

[14] Y. Zhang, "A new merging method and its spectral and spatial effects," Int. J. Remote Sens., vol. 20, pp. 2003-2014, May 1999.

[15] T. A. Wilson, S. K. Rogers, and M. Kabrisky, "Perceptual-based image fusion for hyperspectral data," IEEE Trans. Geosci. Remote Sensing, vol. 35, pp. 1007-1017, July 1997.

[16] B. Aiazzi, L. Alparone, S. Baronti, and A. Garzelli, "Context-driven fusion of high spatial and spectral resolution images based on oversampled multiresolution analysis," IEEE Trans. Geosci. Remote Sensing, vol. 40, pp. 2300-2312, Oct. 2002.

[17] J. Nuñez, X. Otazu, O. Fors, A. Prades, V. Palà, and Romàn Arbiol, "Multiresolution-based image fusion with additive wavelet decomposition," IEEE Trans. Geosci. Remote Sensing, vol. 37, pp. 1204-1211, May 1999

[18] Y. Chibani and A. Houacine, "The joint use of IHS transform and redundant wavelet decomposition for fusing multispectral and panchromatic images," Int. J. Remote Sensing, vol. 23, pp. 3821-3833, Sep. 2002.

[19] S. G. Mallat, "A theory for multiresolution signal decomposition: tThe wavelet representation," IEEE Trans. Pattern Anal. Machine Intell., vol. 11, pp. 674-693, July 1989.

[20] L. Wald, T. Ranchin, and M. Mangolini, "Fusion of satellite images of different spatial resolution: Assessing the quality of resulting images," Photogramm. Eng. Remote Sens., vol. 63, pp. 691-699, June 1997.

[21] P. S. Chavez and A. Y. Kwarteng, "Extracting spectral contrast in Landsat Thematic Mapper image data using selective principal component analysis," Photogramm. Eng. Remote Sens., vol. 55, pp. 339-348, Mar. 1989.

[22] C. Pohl and J. L. Van Genderen, "Multisensor image fusion in remote sensing: Concepts, methods and applications," Int. J. Remote Sens., vol. 19, pp. 823-854, Mar. 1998.

[23] A. R. Smith, "Color gamut transform pairs," Comput. Graph., vol. 12, pp. 12-19, Aug. 1978. 
[24] ACM, "Status report of the graphics standard planning committee," Comput. Graph., vol. 13, no. 3, 1979.

[25] E. M. Schetselaar, "Fusion by the IHS transform: Should we use cylindrical or spherical coordinates?," Int. J. Remote Sens., vol. 19, pp. 759-765, Feb. 1998.

[26] Y. Chibani and A. Houacine, "Fusion of multispectral and radar image in the redundant wavelet domain," Proc. SPIE, vol. 3500, pp. 330-338, Sept. 1998.

[27] S. Mallat, "Multiresolution approximation and wavelet orthonormal bases of L2(R)," Trans. Amer. Math. Soc., vol. 315, no. 1, pp. 69-87, Sept. 1989.

[28] Y. Meyer, Ondelettes et Opérateurs 1: Ondelettes. Paris, France: Hermann, 1990.

[29] I. Daubechies, Ten Lectures on Wavelets. Philadelphia, PA: SIAM, 1992

[30] M. Lang, H. Guo, J. E. Odegard, and C. S. Burrus, "Nonlinear processing of a shift invariant DWT for noise reduction," Proc. SPIE, vol. 2491, pp. 640-651, 1995.

[31] H. Guo, "Theory and applications of the shift-invariant, time varying and undecimated wavelet transforms," M.S. thesis, Rice Univ., Houston, TX, May 1995.

[32] I. Daubechies, "Orthonormal basis of compactly supported wavelets," Commun. Pure Appl. Math., vol. 41, pp. 909-996, Nov. 1988.

[33] M. Mangolini, T. Ranchin, and L. Wald, "Fusion d'images SPOT multispectrale (XS) et panchromatique (P), et d'images radar," in Actes du colloque SPOT-ERS: De l'optique au radar. Les applications de SPOT et ERS, 1993, pp. 199-209.

[34] D. A. Yocky, "Multiresolution wavelet decomposition image merger of Landsat Thematic Mapper and SPOT panchromatic data," Photogramm. Eng. Remote Sens., vol. 62, pp. 1067-1074, Sept. 1996.

[35] L. Wald, "Quality of high resolution synthesized images: Is there a simple criterion?," in Proc. Int. Conf. Fusion Earth Data, Jan. 2000, pp. 99-105.

[36] M. González-Audicana, "Fusión de imágenes multiespectrales y pancromáticas: Desarrollo, aplicación y comparación de diferentes procedimientos. Utilidad de las imágenes resultantes para la discriminación de cultivos en áreas de regadío de Navarra," Eng. Doctoral thesis, Univ. Pública de Navarra, Navarra, Spain, April 2002.

[37] M. González-Audícana, X. Otazu, O. Fors, R. García, and J. Núñez, "Fusion of different spatial and spectral resolution images: Development, application and comparison of new methods based on wavelets," in Proc. Int. Symp. Recent Advances Quantitative Remote Sensing, Sept. 2002, pp. 228-237.

[38] D. A. Yocky, “Artifacts in wavelet image merging," Opt. Eng., vol. 53, pp. 2094-2101, July 1995.

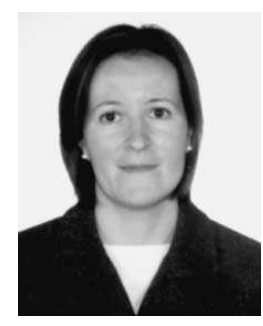

María González-Audícana received the B.S. and Ph.D. degrees in agricultural engineering, with a thesis based on image-fusion, from the Public University of Navarre, Pamplona, Spain, in 1996 and 2001, respectively.

Since 1997, she has been an Assistant Professor with the Department of Projects and Rural Engineering, Public University of Navarre, where she is currently involved in research activities on multisensor data fusion, multispectral image segmentation, and image classification for agricultural

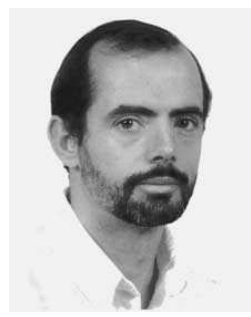

José Luis Saleta received the B.S. degree in forestry engineering from the Polytechnic University of Madrid, Madrid, Spain, in 1988 and the M.A. degree in geography from the University of California, Santa Barbara, in 1995.

From 1995 to 2000, he was an Assistant Professor in the Department of Projects and Rural Engineering, Public University of Navarre, Pamplona, Spain. In 2001, he became a Development Engineer with the MicroEnvironmental Imaging and Analysis Facility, Donald Bren School of Environmental Science and Management, University of California, Santa Barbara.

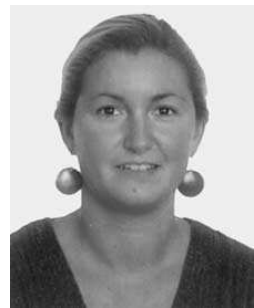

Raquel García Catalán received the B.S. degree in mathematics in 1994 and the Ph.D. degree in mathematical analysis in 1997, both from the University of Zaragoza, Zaragoza, Spain. Her thesis was based on wavelet theory and tight frames.

From 1994 to 1999, she was an Assistant Professor with the University of Zaragoza. During this period, she worked at the University of Seville on a project of image recognizing and then stayed with the Polytechnic University of Torino for six months with an European grant on wavelet theory and numerical analysis. Since 1999, she has been a Professor with the Department of Mathematics and Informatics, Public University of Navarre, Pamplona, Spain. Her current research activity involves multirresolution analysis, frames, and Shannon sampling theory.

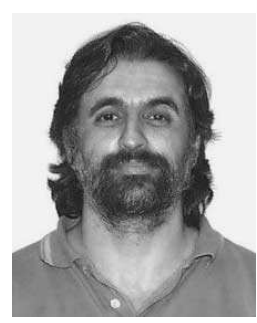

Rafael García received the B.S. degree in agricultural engineering from the University of Córdoba, Córdoba, Spain, in 1985, and the Ph.D. degree in agricultural engineering, from the Polytechnic University of Madrid, Madrid, Spain, in 1995.

Since 1990, he has been a Professor with the Projects and Rural Engineering Department, Public University of Navarre, Pamplona, Spain. 